\title{
Reflets
}

Revue d'intervention sociale et communautaire

\section{Pont entre la culture communautaire et la culture institutionnelle : grille d'analyse organisationnelle pour faciliter l'évaluation de nos actions}

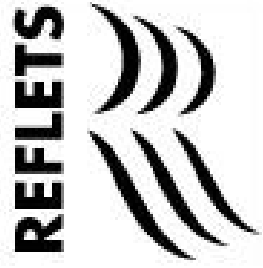

Joscelyne Levesque

Volume 18, numéro 1, printemps 2012

URI : https://id.erudit.org/iderudit/1012334ar

DOI : https://doi.org/10.7202/1012334ar

Aller au sommaire du numéro

Éditeur(s)

Reflets, Revue d'intervention sociale et communautaire

ISSN

1203-4576 (imprimé)

1712-8498 (numérique)

Découvrir la revue

Citer cet article

Levesque, J. (2012). Pont entre la culture communautaire et la culture institutionnelle : grille d'analyse organisationnelle pour faciliter l'évaluation de nos actions. Reflets, 18(1), 120-139. https://doi.org/10.7202/1012334ar d'utilisation que vous pouvez consulter en ligne. 


\section{Pont entre la culture}

communautaire et la culture institutionnelle : grille d'analyse organisationnelle pour faciliter l'évaluation de nos actions

\section{Joscelyne Levesque}

Coordonnatrice de la formation pratique, École de service social, Université d'Ottawa

\section{Introduction}

Dans La société malade de la gestion, Vincent de Gaulejac (2005) présente cette dernière comme une idéologie favorisant le pouvoir économique au détriment du bien commun. Depuis les vingt dernières années, le milieu communautaire est fortement invité à intégrer dans ses pratiques la culture de performance et de compétition propre au pouvoir économique. Souvent, ces demandes s'inscrivent dans une logique administrative qui ne tient que peu compte de la culture particulière au milieu communautaire, culture basée, entre autres, sur l'action citoyenne, la justice sociale, la démocratie et le respect des droits individuels et collectifs. Ces valeurs s'enracinent dans les différentes sphères de l'organisme : dans sa mission, sa vie associative, son ancrage dans la communauté, la représentativité de ses membres au sein des instances décisionnelles, ses pratiques d'action ainsi que dans ses ressources. 
À titre d'exemple, soulignons les formulaires des demandes de subvention et de reddition de compte souvent prescrits et empreints des valeurs managériales dominantes, lesquelles s'éloignent des valeurs démocratiques mises de l'avant par le milieu communautaire. Ne pouvant faire fi de ces demandes, nous présentons dans cet article la Grille d'analyse organisationnelle (voir en annexe) développée à partir des réalités propres au milieu communautaire. Cette grille permet de tracer un portrait d'ensemble de l'organisation tout en respectant la philosophie de l'action communautaire. Développée dans un langage approprié aux valeurs communautaires, elle facilite l'appropriation d'éléments de gouvernance dans un contexte respectant la culture propre au milieu. Elle devient donc un outil soutenant la mission de l'organisme et non pas une fin en soi.

Riche d'une expérience de plus de vingt ans comme directrice en milieu communautaire, nous avons voulu par cet exercice nous doter d'un outil d'appréciation organisationnelle représentant l'essence des actions des organismes communautaires, tout en répondant aux attentes des différents bailleurs de fonds et partenaires gouvernementaux ou institutionnels. Il est parfois essentiel de comprendre le langage de l'autre tout en respectant son propre langage, et ce, afin de bien s'entendre. Nous préférons nous approprier un outil correspondant à nos valeurs plutôt que nous en faire imposer un dans lequel nous ne nous retrouvons pas idéologiquement.

Cette grille d'analyse organisationnelle se veut donc un outil de base pouvant être adapté selon les besoins de l'organisme, comme tout outil de gestion répondant à des besoins et non à une finalité.

Dans La pratique de l'action communautaire autonome (2010), Henri Lamoureux pose la question à savoir si le milieu communautaire est en voie de passer de réformateur social à gestionnaire de problèmes sociaux? La réponse à cette question demeure au cœur de la réalité actuelle et à venir du mouvement communautaire. Afin de garder le cap sur notre action visant la justice sociale, nous devons nous approprier des outils qui, tout en répondant aux exigences des bailleurs de fonds et de certains 
de nos partenaires, respectent notre culture et notre raison d'être. C'est dans cette perspective qu'a été développée cette Grille d'analyse organisationnelle.

\section{Définition des catégories}

Le postulat de base qui a servi de trame de fond pour l'élaboration de la grille reconnait et réaffirme l'existence de pratiques organisationnelles propres au milieu communautaire. Elles s'inspirent de valeurs de justice sociale et d'équité dans lesquelles les liens sociaux occupent une place prépondérante. Elles favorisent la défense des droits individuels et collectifs à travers des actions citoyennes visant le changement social pour le mieuxêtre de toutes et tous. Ces pratiques et valeurs transcendent les activités quotidiennes de l'organisme tant dans sa gestion humaine des ressources que dans la répartition égalitaire du pouvoir entre l'ensemble des membres y œuvrant : entre autres, participation aux instances formelles de décision - assemblée générale annuelle, conseil d'administration, comités de travail - mise en place de lieux informels de prise de parole, implication dans l'organisation de différentes activités et participation au processus d'appréciation des activités (Duval, et collab., 2005).

Afin d'optimiser l'utilisation de la grille d'analyse organisationnelle, il nous apparait important de définir les dix catégories qui y figurent.

\section{Mission et valeurs (finalité et sens donné aux actions)}

La mission est à la base même de la naissance d'un organisme. C'est l'élément qui définit son identité. C'est aussi autour de la mission que se mobilisent les personnes qui se sentent interpellées par la raison d'être de l'organisme.

Les valeurs, quant à elles, représentent le sens donné aux pratiques de l'organisme. Elles teintent fortement les actions et services offerts. Elles définissent en sorte les couleurs de l'organisme. 
2. Vie associative (processus décisionnel démocratique, exercice de la citoyenneté, implication des membres de la communauté, etc.)

Les organismes communautaires peuvent compter sur différentes instances démocratiques pour vivifier leur vie associative : entre autres, assemblée générale annuelle, conseil d'administration et action citoyenne à travers l'implication bénévole. Ces instances sont essentielles pour favoriser l'implication des membres de l'organisme à différents niveaux afin de développer chez eux un sentiment d'appartenance à la collectivité et ainsi favoriser de leur part l'exercice de la démocratie.

\section{Conseil d'administration}

Le conseil d'administration met de l'avant toute la question de la représentation citoyenne. Sa composition et son rôle varient d'un organisme à un autre. Pour qu'il exerce son rôle d'espace citoyen, il doit demeurer accessible aux personnes fréquentant l'organisme et aux citoyennes et citoyens qui se sentent interpellés par la mission. C'est un lieu d'apprentissage riche pour toute la question de l'espace démocratique et de l'exercice du pouvoir.

\section{Ancrage dans le milieu et réseautage (qualité des liens} entre l'organisme et ses partenaires)

Un organisme communautaire ne peut agir seul. Il fait partie d'un réseau composé tant de personnes que d'organisations qui se sentent interpellées par toute la question de la justice sociale et du respect des droits individuels et collectifs. Les relations avec ces partenaires sont pour l'organisme gages de succès dans ses efforts visant à améliorer la qualité de vie individuelle et collective des citoyennes et citoyens. Une des forces du milieu communautaire est sa capacité de créer des liens significatifs avec l'ensemble de ses partenaires et constituer une force vive au nom de la justice sociale.

\section{Santé financière}

Tout en reconnaissant que le financement d'un organisme n'est pas une fin en soi, il n'en demeure pas moins essentiel pour 
la réalisation de sa mission et le respect des valeurs mises de l'avant. Un financement insuffisant met en péril l'autonomie de l'organisme qui devra alors faire des choix difficiles qui parfois ne respectent pas les valeurs prônées tant sur le plan des conditions de travail que sur celui des activités.

\section{Pratiques}

Les pratiques sont le reflet de la mission et des valeurs. En ce sens, elles doivent mettre de l'avant des activités favorisant l'expression citoyenne. C'est donc un lieu où se côtoient les dimensions personnelles et sociales, où vont de pair l'accompagnement individuel et l'action politique et où les activités mises en place sont définies à partir du respect des droits des personnes et de la société.

\section{Ressources humaines}

Les membres de l'équipe, tant bénévoles que rémunérés, sont au cœur de l'action de l'organisme. Plus que de simples "ressources ", ils deviennent les agents multiplicateurs de la mission de l'organisme au sein de la société. Il est donc primordial qu'ils soient impliqués à tous les niveaux de l'organisme, tant dans la prise de décision que dans la définition et l'actualisation des actions porteuses de la mission.

\section{Stratégie et planification}

Le milieu communautaire possède une forte tradition orale. L'histoire se crée au fil des jours et peu d'écrits en illustrent le cheminement. Il est donc important de maintenir cette tradition orale tout en développant un minimum d'écrits permettant d'illustrer les actions passées et à venir de l'organisme. Les notions de stratégie et de planification permettent d'identifier les éléments importants afin de bien cerner les actions à venir et ainsi maximiser l'énergie de l'équipe.

\section{Leadership de la direction générale ou coordination}

La direction générale et les coordinations des différents secteurs de l'organisme sont des éléments clés au sein d'une organisation. Ils sont porteurs de la mission et des valeurs de l'organisme. 


\section{Attitudes}

Les attitudes reflètent les valeurs prônées par l'organisme et se manifestent à travers les comportements des membres impliqués au sein de l'organisme. C'est souvent en regardant les membres agir que l'on peut apprécier le climat de travail au sein de l'équipe.

\section{Pistes pour l'utilisation de la grille d'analyse organisationnelle}

Ces dix catégories sont donc à apprécier dans la Grille d'analyse organisationnelle proposée. Elles représentent, selon nous, les principaux secteurs organisationnels d'un organisme communautaire. L'appréciation de ces secteurs permettra de tracer un portrait de sa vitalité.

En se servant des rapports d'activité, des procès-verbaux des réunions de l'équipe et de celles du conseil d'administration, d'échanges avec les personnes clés de l'organisme, un comité composé de représentants des différents groupes œuvrant au sein de l'organisme évalue chaque catégorie selon les éléments décrits. Une fois le tableau de chaque catégorie rempli, il suffit d'en reproduire le pointage à l'intérieur des deux derniers tableaux. Cela permet de faire la synthèse de l'appréciation et d'obtenir une vue d'ensemble des forces et limites organisationnelles de l'organisme.

Par la suite, les sections analysées permettent, si souhaité, d'identifier les secteurs où l'organisme aimerait effectuer des changements. Ces derniers peuvent s'inscrire à l'intérieur du plan d'action pour l'année en cours ou l'année suivante.

Nous vous invitons à adapter à vos besoins les différents éléments composant la grille. En fait, nous souhaitons que vous vous appropriiez le contenu de la grille afin qu'il reflète votre propre culture organisationnelle ou celle d'une autre organisation. Le milieu communautaire n'est pas homogène. Ce qui fait sa 
richesse, c'est sa grande diversité qui représente bien les citoyennes et citoyens qui s'y impliquent.

Une fois la grille d'analyse organisationnelle remplie, il est important, dans un premier temps, de tracer un portrait d'ensemble des principaux éléments qui ressortent tant sur le plan des forces que sur celui des faiblesses identifiées. Par exemple, la grille identifie une vie associative très présente et active, mais une gestion humaine des ressources quelque peu négligée, ou encore, un conseil d'administration omniprésent au sein de la gestion quotidienne et une direction générale davantage impliquée auprès des partenaires qu'auprès de l'équipe de travail. Ces deux exemples méritent une discussion de fond au sein du conseil d'administration et avec les membres de l'organisation afın d'évaluer les actions à poser selon la philosophie et la vision préconisée au sein de l'organisme.

Cette discussion peut avoir lieu lors de la journée consacrée au bilan annuel afin d'inclure dans le plan d'action les moyens qui seront mis de l'avant pour améliorer les éléments retenus. Ou encore, un comité de travail peut être mis sur pied afin de proposer au conseil d'administration de se pencher sur certains éléments jugés importants par l'organisation.

La grille permet aussi de souligner les forces de l'organisation, lesquelles pourraient être mentionnées lors du bilan annuel et — pourquoi pas? — dans le rapport annuel d'activité. Souvent, les points à améliorer sont davantage mis de l'avant que les bons coups réalisés au cours de l'année.

Parfois, l'analyse de la grille soulève des perceptions différentes entre les groupes impliqués au sein de l'organisation. Un groupe peut évaluer que la vie associative occupe une place importante alors qu'un autre peut trouver que la vie associative n'est pas assez développée. Les deux points de vue ont leur importance et nous devons nous attarder sur les facteurs pouvant expliquer ces différences et évaluer s'il y a lieu de poser une action.

Enfin, il est important de souligner que le portrait qui se dégage de la grille n'est pas statique. C'est une photo prise à un moment donné au cours de la vie de l'organisation. Une seconde 
photo prise l'année suivante peut donner des résultats similaires ou différents selon l'évolution de l'organisation.

\section{Conclusion}

Dans un monde du travail axé sur la compétition et la performance et où la culture de la gestion est omniprésente, nous pouvons, si souhaité, rejeter tout ce qui touche le pouvoir managérial. De notre côté, comme directrice d'organismes communautaires, nous avons choisi de nous approprier les outils développés dans le monde de la gestion et de les adapter dans un langage qui rejoint les valeurs communautaires et selon une perspective favorisant la répartition du pouvoir et l'exercice de la citoyenneté. Notre pratique nous permet d'affirmer que ces outils facilitent nos actions collectives et favorisent une meilleure compréhension du langage de nos partenaires gouvernementaux, dont les bailleurs de fonds et les milieux institutionnels.

\section{Bibliographie}

DE GAULEJAC,Vincent (2005). La société malade de la gestion, Paris, Éditions du Seuil, 275 p.

DUVAL Michelle, et collab. (2004). Les organismes au Québec : pratiques et enjeux, Boucherville, Gaëtan Morin éditeur, $176 \mathrm{p}$.

LAMOUREUX, Henri (2010). La pratique de l'action communautaire autonome, Québec, Presses de l’Université du Québec, 132 p.

SHRAGGE,Éric (2006). Action communautaire : dérives et possibles, Montréal, Les Éditions Écosociété, $242 \mathrm{p}$. 


\section{GRILLE D'ANALYSE ORGANISATIONNELLE}

Cette grille d'analyse organisationnelle a pour but de tracer un portrait sommaire des forces et des limites d'une organisation communautaire. Suite à vos observations et à vos discussions avec les gens clés de l'organisme, veuillez apprécier de 1 à 3 la force de chacune des composantes.

\begin{tabular}{|c|c|c|}
\hline \multicolumn{2}{|c|}{$\begin{array}{l}\text { MISSION ET VALEURS } \\
\text { (finalité et sens aux actions/activités) }\end{array}$} & \multirow{2}{*}{$\begin{array}{l}\text { Total de points : } \\
(\max .21 \text { points })\end{array}$} \\
\hline FORT (3) & ADÉQUAT (2) & \\
\hline $\begin{array}{l}\text { La mission de l'organisme } \\
\text { est définie selon les besoins/ } \\
\text { intérêts de la communauté. }\end{array}$ & $\begin{array}{l}\text { La mission de l'organisme est } \\
\text { définie à partir des besoins } \\
\text { identifiés par certains acteurs } \\
\text { et actrices du milieu. }\end{array}$ & $\begin{array}{l}\text { La mission de l'organisme } \\
\text { est mal définie et couvre de } \\
\text { multiples champs d'action. }\end{array}$ \\
\hline $\begin{array}{l}\text { La mission de l'organisme } \\
\text { vise le changement social et la } \\
\text { justice sociale. }\end{array}$ & $\begin{array}{l}\text { La mission de l'organisme vise } \\
\text { la justice sociale. }\end{array}$ & $\begin{array}{l}\text { La mission de l'organisme } \\
\text { vise majoritairement la } \\
\text { dispensation de services. }\end{array}$ \\
\hline $\begin{array}{l}\text { Les membres de l'organisme } \\
\text { connaissent bien sa mission. }\end{array}$ & $\begin{array}{l}\text { Les membres du CA et de } \\
\text { l'équipe connaissent la mission } \\
\text { de l'organisme. }\end{array}$ & $\begin{array}{l}\text { La mission est peu connue } \\
\text { des membres du CA et de } \\
\text { l'équipe. }\end{array}$ \\
\hline $\begin{array}{l}\text { Les membres connaissent bien } \\
\text { l'historique de l'organisme. }\end{array}$ & $\begin{array}{l}\text { Les membres du CA et } \\
\text { de l'équipe connaissent } \\
\text { l'historique de l'organisme. }\end{array}$ & $\begin{array}{l}\text { L'histoire de l'organisme s'est } \\
\text { perdue au fil des ans. }\end{array}$ \\
\hline $\begin{array}{l}\text { Les valeurs de l'organisme } \\
\text { sont bien connues } \\
\text { des membres et ils s'y } \\
\text { reconnaissent. }\end{array}$ & $\begin{array}{l}\text { Les valeurs de l'organisme } \\
\text { sont connues du CA et de } \\
\text { l'équipe. }\end{array}$ & $\begin{array}{l}\text { Les valeurs de l'organisme } \\
\text { sont mal définies. }\end{array}$ \\
\hline $\begin{array}{l}\text { L'organisme démontre une } \\
\text { bonne capacité à se remettre } \\
\text { en question et à adapter ses } \\
\text { pratiques selon les besoins/ } \\
\text { intérêts exprimés par les } \\
\text { personnes participantes }\end{array}$ & $\begin{array}{l}\text { L'organisme peut se remettre } \\
\text { en question et adapter ses } \\
\text { pratiques pourvu que les } \\
\text { ressources financières et } \\
\text { humaines soient disponibles. }\end{array}$ & $\begin{array}{l}\text { L'organisme démontre peu } \\
\text { d'ouverture à remettre en } \\
\text { question sa mission et ses } \\
\text { valeurs. }\end{array}$ \\
\hline $\begin{array}{l}\text { L'organisme fait preuve d'un } \\
\text { grand potentiel d'innovation } \\
\text { et de créativité dans la mise en } \\
\text { œuvre de sa mission }\end{array}$ & $\begin{array}{l}\text { L'organisme est ouvert aux } \\
\text { idées innovantes et créatives } \\
\text { dans la mise en œuvre de sa } \\
\text { mission. }\end{array}$ & $\begin{array}{l}\text { L'organisme démontre peu } \\
\text { d'ouverture face aux idées } \\
\text { nouvelles et créatrices dans la } \\
\text { mise en œuvre de sa mission. }\end{array}$ \\
\hline Nombre de points : & Nombre de points : & Nombre de points : \\
\hline
\end{tabular}




\begin{tabular}{|c|c|c|}
\hline \multicolumn{2}{|c|}{$\begin{array}{l}\text { VIE ASSOCIATIVE } \\
\text { (processus décisionnel démocratique, exercice de la } \\
\text { citoyenneté, implication de membres de la communauté, etc.) }\end{array}$} & $\begin{array}{l}\text { Total de points }: \\
\text { (max. } 21 \text { points) }\end{array}$ \\
\hline FORT (3) & ADÉQUAT (2) & FAIBLE (1) \\
\hline $\begin{array}{l}\text { Processus démocratique solide, } \\
\text { très bien établi (présence } \\
\text { des personnes participantes, } \\
\text { travailleuses et bénévoles } \\
\text { dans l'ensemble des sphères } \\
\text { décisionnelles de l'organisme). }\end{array}$ & $\begin{array}{l}\text { Processus démocratique établi } \\
\text { et opérationnel (présence } \\
\text { des personnes participantes, } \\
\text { travailleuses et bénévoles } \\
\text { au sein de quelques sphères } \\
\text { décisionnelles de l'organisme). }\end{array}$ & $\begin{array}{l}\text { Processus démocratique } \\
\text { mal défini (absence des } \\
\text { personnes participantes et des } \\
\text { bénévoles au sein des sphères } \\
\text { décisionnelles de l'organisme). }\end{array}$ \\
\hline $\begin{array}{l}\text { Prise en charge collective } \\
\text { des activités (implication } \\
\text { des personnes participantes, } \\
\text { bénévoles et travailleuses dans } \\
\text { l'identification des besoins/ } \\
\text { intérêts et des activités mises } \\
\text { en place). }\end{array}$ & $\begin{array}{l}\text { Prise en charge collective } \\
\text { des activités à quelques } \\
\text { niveaux (implication des } \\
\text { personnes participantes, } \\
\text { bénévoles et travailleuses dans } \\
\text { l'identification des besoins/ } \\
\text { intérêts et des activités mises } \\
\text { en place). }\end{array}$ & $\begin{array}{l}\text { Axé davantage sur la prise } \\
\text { en charge individuelle } \\
\text { des activités (absence des } \\
\text { personnes participantes et des } \\
\text { bénévoles dans l'identification } \\
\text { des besoins/intérêts et des } \\
\text { activités mises en place). }\end{array}$ \\
\hline $\begin{array}{l}\text { Primauté du processus sur } \\
\text { l'atteinte des résultats. }\end{array}$ & $\begin{array}{l}\text { Le processus est important, } \\
\text { mais les résultats tout autant. }\end{array}$ & $\begin{array}{l}\text { Prépondérance des résultats } \\
\text { sur le processus. }\end{array}$ \\
\hline $\begin{array}{l}\text { Favorise l'empowerment } \\
\text { collectif (être propriétaire } \\
\text { collectivement d'un lac } \\
\text { poissonneux). }\end{array}$ & $\begin{array}{l}\text { Présence de la philosophie } \\
\text { d'empowerment (apprendre à } \\
\text { pêcher). }\end{array}$ & $\begin{array}{l}\text { Philosophie caritative (donner } \\
\text { du poisson). }\end{array}$ \\
\hline $\begin{array}{l}\text { Actions orientées vers le } \\
\text { changement social et la justice } \\
\text { sociale. }\end{array}$ & $\begin{array}{l}\text { Actions orientées vers la } \\
\text { justice sociale, mais très peu } \\
\text { vers le changement social. }\end{array}$ & $\begin{array}{l}\text { Actions centrées sur les } \\
\text { services. }\end{array}$ \\
\hline $\begin{array}{l}\text { Présence marquante de } \\
\text { bénévoles au sein des activités } \\
\text { de l'organisme. }\end{array}$ & $\begin{array}{l}\text { Présence de bénévoles au } \\
\text { sein de certaines activités de } \\
\text { l'organisme. }\end{array}$ & $\begin{array}{l}\text { Peu ou pas de bénévoles } \\
\text { impliqués. }\end{array}$ \\
\hline $\begin{array}{l}\text { Taux de participation } \\
\text { significatif et diversifié à } \\
\text { l'AGA (plus de } 30 \text { personnes). }\end{array}$ & $\begin{array}{l}\text { Taux de participation } \\
\text { satisfaisant à l'AGA ( } 20 \\
\text { personnes et moins). }\end{array}$ & $\begin{array}{l}\text { Faible taux de participation à } \\
\text { l'AGA (personnel et membres } \\
\text { du CA). }\end{array}$ \\
\hline Nombre de points : & Nombre de points : & Nombre de points : \\
\hline
\end{tabular}




\begin{tabular}{|l|l|l|}
\hline \multicolumn{2}{|l|}{ CONSEIL D'ADMINISTRATION } & $\begin{array}{l}\text { Total de points : } \\
\text { (max. 21 points) }\end{array}$ \\
\hline FORT (3) & ADÉQUAT (2) & FAIBLE (1) \\
\hline $\begin{array}{l}\text { Les membres du CA ont } \\
\text { un intérêt marqué pour } \\
\text { la mission de l'organisme } \\
\text { (engagement personnel et } \\
\text { collectif). }\end{array}$ & $\begin{array}{l}\text { Les membres du CA } \\
\text { connaissent bien la mission de } \\
\text { l'organisme. }\end{array}$ & $\begin{array}{l}\text { Certains membres du CA } \\
\text { connaissent la mission de } \\
\text { l'organisme. }\end{array}$ \\
\hline $\begin{array}{l}\text { Bonne connaissance des } \\
\text { dossiers politiques (lecture de } \\
\text { changement social et justice } \\
\text { sociale). }\end{array}$ & $\begin{array}{l}\text { Bien au fait des dossiers et des } \\
\text { enjeux qui en découlent. }\end{array}$ & $\begin{array}{l}\text { Membres du CA sont } \\
\text { relativement au courant des } \\
\text { enjeux politiques des dossiers. }\end{array}$ \\
\hline $\begin{array}{l}\text { Expertise des membres est } \\
\text { diversifiée et pertinente à la } \\
\text { mission de l'organisme. }\end{array}$ & $\begin{array}{l}\text { Expertise pertinente et intérêt } \\
\text { manifeste pour la mission de } \\
\text { l'organisme. }\end{array}$ & $\begin{array}{l}\text { Membres du CA sont } \\
\text { relativement impliqués. }\end{array}$ \\
\hline $\begin{array}{l}\text { Des sièges au sein du CA } \\
\text { sont réservés aux personnes } \\
\text { participantes. }\end{array}$ & $\begin{array}{l}\text { Un siège est réservé à un } \\
\text { membre travailleur. }\end{array}$ & $\begin{array}{l}\text { Aucun siège n'est réservé } \\
\text { à un membre travailleur ou } \\
\text { personne participants. }\end{array}$ \\
\hline $\begin{array}{l}\text { Majorité des membres du CA } \\
\text { sont issus de la communauté } \\
\text { visée par la mission. }\end{array}$ & $\begin{array}{l}\text { Un certain nombre des } \\
\text { membres du CA sont issus de } \\
\text { la communauté visée par la } \\
\text { mission. }\end{array}$ & $\begin{array}{l}\text { Proviennent de différents } \\
\text { milieux (partenaires, } \\
\text { communauté, etc.). }\end{array}$ \\
\hline $\begin{array}{l}\text { Bon soutien à la direction et } \\
\text { à l'équipe. }\end{array}$ & $\begin{array}{l}\text { Bonne complémentarité des } \\
\text { rôles CA et direction. }\end{array}$ & $\begin{array}{l}\text { Méconnaissance des rôles du } \\
\text { CA et de la direction. }\end{array}$ \\
\hline $\begin{array}{l}\text { Réunions régulières et } \\
\text { efficaces où les sujets à l'ordre } \\
\text { du jour sont pertinents et la } \\
\text { durée des réunions ne dépasse } \\
\text { pas deux heures. }\end{array}$ & $\begin{array}{l}\text { Réunions régulières où les } \\
\text { sujets à l'ordre du jour sont } \\
\text { plus ou moins pertinents et la } \\
\text { durée des réunions ne dépasse } \\
\text { pas trois heures. }\end{array}$ & $\begin{array}{l}\text { Rencontres peu fréquentes et } \\
\text { absence de suivi des dossiers. } \\
\text { Réunions d'une durée variée. }\end{array}$ \\
\hline Nombre de points : & Nombre de points : & Nombre de points : \\
\hline
\end{tabular}




\begin{tabular}{|c|c|c|}
\hline \multicolumn{2}{|c|}{$\begin{array}{l}\text { ANCRAGE DANS LE MILIEU ET RÉSEAUTAGE } \\
\text { (qualité des liens entre l'organisme et ses partenaires) }\end{array}$} & \multirow{2}{*}{$\begin{array}{l}\text { Total de points : } \\
\text { (max. } 21 \text { points) } \\
\text { FAIBLE }(\mathbf{1})\end{array}$} \\
\hline FORT (3) & ADÉQUAT (2) & \\
\hline $\begin{array}{l}\text { Fort soutien de la } \\
\text { communauté locale et/ } \\
\text { ou régionale (présence des } \\
\text { membres de la communauté } \\
\text { au sein du CA ou des } \\
\text { comités). }\end{array}$ & $\begin{array}{l}\text { Intérêt démontré par la } \\
\text { communauté locale ou } \\
\text { régionale. }\end{array}$ & $\begin{array}{l}\text { Démonstration d'un certain } \\
\text { leadership ponctuel dans son } \\
\text { milieu. }\end{array}$ \\
\hline $\begin{array}{l}\text { Forte présence des } \\
\text { partenaires du milieu } \\
\text { au sein des différentes } \\
\text { instances de l'organisme } \\
\text { (comité de sélection, comité } \\
\text { d'appréciation des actions de } \\
\text { l'organisme). }\end{array}$ & $\begin{array}{l}\text { Appui du milieu par la } \\
\text { participation à des comités } \\
\text { ponctuels. }\end{array}$ & $\begin{array}{l}\text { Appui moral des partenaires, } \\
\text { mais peu d'implication au sein } \\
\text { des comités (lettres d'appui). }\end{array}$ \\
\hline $\begin{array}{l}\text { Différentes sources de } \\
\text { financement (plus que trois } \\
\text { bailleurs de fonds). }\end{array}$ & $\begin{array}{l}\text { Présence de bailleurs de fonds } \\
\text { (trois et moins). }\end{array}$ & $\begin{array}{l}\text { Présence timide de quelques } \\
\text { bailleurs de fonds. }\end{array}$ \\
\hline $\begin{array}{l}\text { Grande diversité de } \\
\text { partenariats. }\end{array}$ & Diversité de partenariats. & $\begin{array}{l}\text { Un à deux partenariats } \\
\text { significatifs. }\end{array}$ \\
\hline $\begin{array}{l}\text { Reconnaissance de } \\
\text { l'expertise et des actions de } \\
\text { l'organisme dans son milieu } \\
\text { d'appartenance. }\end{array}$ & $\begin{array}{l}\text { L'organisme est reconnu dans } \\
\text { son milieu. }\end{array}$ & $\begin{array}{l}\text { L'organisme est peu connu } \\
\text { dans son milieu (besoin } \\
\text { d'expliquer sa mission). }\end{array}$ \\
\hline $\begin{array}{l}\text { Grande capacité de } \\
\text { mobilisation des partenaires } \\
\text { par l'organisme. }\end{array}$ & $\begin{array}{l}\text { Capacité de l'organisme à } \\
\text { susciter la participation des } \\
\text { partenaires. }\end{array}$ & $\begin{array}{l}\text { Implication ponctuelle des } \\
\text { partenaires et à court terme. }\end{array}$ \\
\hline $\begin{array}{l}\text { L'organisme est une référence } \\
\text { au plan du leadership et de } \\
\text { l'excellence de sa gestion. On } \\
\text { cherche à apprendre d'eux. }\end{array}$ & $\begin{array}{l}\text { L'organisme est respecté sur le } \\
\text { terrain. }\end{array}$ & $\begin{array}{l}\text { L'organisme semble être évité } \\
\text { sur le terrain. }\end{array}$ \\
\hline Nombre de points : & Nombre de points : & Nombre de points : \\
\hline
\end{tabular}




\begin{tabular}{|c|c|c|}
\hline \multicolumn{2}{|l|}{ SANTÉ FINANCIÈRE } & \multirow{2}{*}{$\begin{array}{l}\begin{array}{l}\text { Total de points : } \\
\text { (max. } 21 \text { points) }\end{array} \\
\text { FAIBLE }(1)\end{array}$} \\
\hline FORT (3) & ADÉQUAT (2) & \\
\hline $\begin{array}{l}\text { L'organisme détient l'appui } \\
\text { de plus que cinq bailleurs de } \\
\text { fonds. }\end{array}$ & $\begin{array}{l}\text { L'organisme détient l'appui } \\
\text { de deux à quatre bailleurs de } \\
\text { fonds. }\end{array}$ & $\begin{array}{l}\text { L'organisme détient l'appui } \\
\text { d'un seul bailleur de fonds. }\end{array}$ \\
\hline $\begin{array}{l}\text { L'organisme fait sur une base } \\
\text { continue de la recherche et du } \\
\text { développement de nouvelles } \\
\text { sources de financement. }\end{array}$ & $\begin{array}{l}\text { L'organisme fait de } \\
\text { la recherche et du } \\
\text { développement de nouvelles } \\
\text { sources de financement } \\
\text { par vagues d'intérêts et de } \\
\text { priorités. }\end{array}$ & $\begin{array}{l}\text { L'organisme ne fait aucune } \\
\text { recherche active de nouvelles } \\
\text { sources de financement. }\end{array}$ \\
\hline $\begin{array}{l}\text { L'organisme a une autonomie } \\
\text { face aux bailleurs de fonds. }\end{array}$ & $\begin{array}{l}\text { L'organisme négocie une } \\
\text { partie de son autonomie avec } \\
\text { les bailleurs de fonds. }\end{array}$ & $\begin{array}{l}\text { L'organisme n'a aucune } \\
\text { autonomie face aux bailleurs } \\
\text { de fonds. }\end{array}$ \\
\hline $\begin{array}{l}\text { L'organisme fait des suivis } \\
\text { continus des prévisions } \\
\text { budgétaires (base mensuelle). }\end{array}$ & $\begin{array}{l}\text { L'organisme fait des suivis } \\
\text { réguliers des prévisions } \\
\text { budgétaires (base } \\
\text { trimestrielle). }\end{array}$ & $\begin{array}{l}\text { L'organisme fait des suivis } \\
\text { sporadiques des prévisions } \\
\text { budgétaires (quelques fois par } \\
\text { année). }\end{array}$ \\
\hline $\begin{array}{l}\text { La comptabilité et la } \\
\text { documentation financière de } \\
\text { l'organisme est à jour (moins } \\
\text { le mois en cours). }\end{array}$ & $\begin{array}{l}\text { La comptabilité et la } \\
\text { documentation financière de } \\
\text { l'organisme sont relativement } \\
\text { à jour (dernier trimestre). }\end{array}$ & $\begin{array}{l}\text { La comptabilité et la } \\
\text { documentation financière de } \\
\text { l'organisme ne sont pas à jour } \\
\text { (trimestre et plus). }\end{array}$ \\
\hline $\begin{array}{l}\text { L'organisme possède des } \\
\text { outils lui permettant } \\
\text { d'exercer un contrôle sur sa } \\
\text { situation financière (processus } \\
\text { d'autorisation des comptes de } \\
\text { dépense, élaboration et suivis } \\
\text { des prévisions budgétaires, } \\
\text { etc.) }\end{array}$ & $\begin{array}{l}\text { L'organisme possède très peu } \\
\text { d'outils de contrôle au niveau } \\
\text { de sa situation financière. }\end{array}$ & $\begin{array}{l}\text { L'organisme ne possède aucun } \\
\text { outil lui permettant d'exercer } \\
\text { un contrôle sur sa situation } \\
\text { financière. }\end{array}$ \\
\hline $\begin{array}{l}\text { L'organisme a bâti une réserve } \\
\text { de fonds de roulement qui } \\
\text { peut les soutenir de } 4 \text { à } 6 \\
\text { mois. }\end{array}$ & $\begin{array}{l}\text { L'organisme a bâti une réserve } \\
\text { de fonds de roulement qui } \\
\text { peut les soutenir de } 1 \text { à } 3 \\
\text { mois. }\end{array}$ & $\begin{array}{l}\text { L'organisme n'a aucune } \\
\text { réserve de fonds de roulement. } \\
\text { L'organisme vit au jour le } \\
\text { jour. }\end{array}$ \\
\hline Nombre de points : & Nombre de points : & Nombre de points : \\
\hline
\end{tabular}




\begin{tabular}{|c|c|c|}
\hline \multicolumn{2}{|l|}{$\begin{array}{l}\text { PRATIQUES } \\
\text { (activités, actions et services) }\end{array}$} & \multirow{2}{*}{$\begin{array}{l}\text { Total de points : } \\
(\max .21 \text { points }) \\
\text { FAIBLE (1) }\end{array}$} \\
\hline FORT (3) & ADÉQUAT (2) & \\
\hline $\begin{array}{l}\text { Les pratiques de l'organisme } \\
\text { favorisent la prise en charge } \\
\text { individuelle et collective des } \\
\text { personnes participantes. }\end{array}$ & $\begin{array}{l}\text { Les pratiques de l'organisme } \\
\text { visent davantage la prise en } \\
\text { charge individuelle et collective } \\
\text { des personnes participantes. }\end{array}$ & $\begin{array}{l}\text { Les pratiques de l'organisme } \\
\text { favorisent la prise en charge } \\
\text { individuelle des personnes } \\
\text { participantes. }\end{array}$ \\
\hline $\begin{array}{l}\text { L'analyse des situations } \\
\text { présentées par les personnes } \\
\text { participantes englobe } \\
\text { l'ensemble des facteurs } \\
\text { sociopolitiques et économiques. }\end{array}$ & $\begin{array}{l}\text { Les pratiques de l'organisme } \\
\text { tentent de mettre de l'avant } \\
\text { les facteurs sociopolitiques et } \\
\text { économiques. }\end{array}$ & $\begin{array}{l}\text { Les pratiques de l'organisme } \\
\text { se limitent aux facteurs } \\
\text { individuels. }\end{array}$ \\
\hline $\begin{array}{l}\text { Les activités/actions mises en } \\
\text { place respectent les limites } \\
\text { humaines et financières de } \\
\text { l'organisme. }\end{array}$ & $\begin{array}{l}\text { Les activités/actions mises } \\
\text { en place ne respectent pas } \\
\text { toujours les limites humaines et } \\
\text { financières de l'organisme. }\end{array}$ & $\begin{array}{l}\text { Les activités/actions mises de } \\
\text { l'avant respectent rarement les } \\
\text { limites humaines et financières } \\
\text { de l'organisme. }\end{array}$ \\
\hline $\begin{array}{l}\text { Les activités/actions de } \\
\text { l'organisme favorisent l'exercice } \\
\text { de la citoyenneté chez ses } \\
\text { membres. }\end{array}$ & $\begin{array}{l}\text { Les activités/actions de } \\
\text { l'organisme tentent de mettre } \\
\text { de l'avant l'exercice de la } \\
\text { citoyenneté. }\end{array}$ & $\begin{array}{l}\text { Les activités/actions de } \\
\text { l'organisme répondent aux } \\
\text { besoins immédiats de la } \\
\text { personne. }\end{array}$ \\
\hline $\begin{array}{l}\text { L'organisme joue un rôle } \\
\text { d'action sociale en menant par } \\
\text { exemple des actions collectives } \\
\text { pour la défense des droits de ses } \\
\text { membres. }\end{array}$ & $\begin{array}{l}\text { L'organisme participe aux } \\
\text { actions collectives organisées } \\
\text { par ses partenaires. }\end{array}$ & $\begin{array}{l}\text { L'organisme participe rarement } \\
\text { aux actions collectives } \\
\text { organisées par ses partenaires. }\end{array}$ \\
\hline $\begin{array}{l}\text { L'organisme est capable de } \\
\text { résister aux pressions exercées } \\
\text { par les demandes de ses } \\
\text { partenaires ou bailleurs de } \\
\text { fonds pour modifier sa mission } \\
\text { ou son rôle. }\end{array}$ & $\begin{array}{l}\text { L'organisme éprouve des } \\
\text { difficultés financières et a de la } \\
\text { difficulté à résister aux pressions } \\
\text { des demandes de ses partenaires } \\
\text { et bailleurs de fonds à modifier } \\
\text { sa mission. }\end{array}$ & $\begin{array}{l}\text { L'organisme a modifié sa } \\
\text { mission et son rôle à la suite des } \\
\text { pressions de ses partenaires ou } \\
\text { bailleurs de fonds. }\end{array}$ \\
\hline $\begin{array}{l}\text { Les actions/activités de } \\
\text { l'organisme reflètent bien } \\
\text { les valeurs organisationnelles } \\
\text { mises de l'avant au sein de } \\
\text { l'organisme. }\end{array}$ & $\begin{array}{l}\text { Les actions/activités de } \\
\text { l'organisme sont habituellement } \\
\text { le reflet des valeurs } \\
\text { organisationnelles mises de } \\
\text { l'avant au sein de l'organisme. }\end{array}$ & $\begin{array}{l}\text { Les valeurs organisationnelles } \\
\text { sont méconnues et absentes des } \\
\text { actions/activités de l'organisme. }\end{array}$ \\
\hline Nombre de points : & Nombre de points : & Nombre de points : \\
\hline
\end{tabular}




\begin{tabular}{|c|c|c|}
\hline \multicolumn{2}{|l|}{ RESSOURCES HUMAINES } & \multirow{2}{*}{$\begin{array}{l}\text { Total de points : } \\
(\max .21 \text { points })\end{array}$} \\
\hline FORT (3) & ADÉQUAT (2) & \\
\hline $\begin{array}{l}\text { L'organisme a une excellente } \\
\text { rétention des membres de } \\
\text { l'équipe ( } 5 \text { ans et plus). }\end{array}$ & $\begin{array}{l}\text { L'organisme a une bonne } \\
\text { rétention des membres de } \\
\text { l'équipe (majorité de } 2 \text { à } 5 \\
\text { ans). }\end{array}$ & $\begin{array}{l}\text { L'organisme a un faible taux } \\
\text { de rétention des membres de } \\
\text { l'équipe ( } 2 \text { ans et moins). }\end{array}$ \\
\hline $\begin{array}{l}\text { Les conditions de travail et } \\
\text { salariales sont supérieures à } \\
\text { celle au sein des organismes } \\
\text { similaires. }\end{array}$ & $\begin{array}{l}\text { Les conditions de travail et } \\
\text { salariales sont équivalentes à } \\
\text { des organismes semblables ou } \\
\text { en progression. }\end{array}$ & $\begin{array}{l}\text { Les conditions de travail et } \\
\text { salariales sont sous la moyenne } \\
\text { des organismes semblables ou } \\
\text { en décroissance. }\end{array}$ \\
\hline $\begin{array}{l}\text { Très faible taux d'épuisement } \\
\text { professionnel ( } 1 \text { situation } \\
\text { au cours des } 3 \text { dernières } \\
\text { années) et très faible taux } \\
\text { d'absentéisme. }\end{array}$ & $\begin{array}{l}\text { Faible taux d'épuisement } \\
\text { professionnel } \\
\text { ( } 2 \text { situation au cours des } 3 \\
\text { dernières années) et faible } \\
\text { taux d'absentéisme. }\end{array}$ & $\begin{array}{l}\text { Taux d'épuisement } \\
\text { professionnel élevé ( } 3 \\
\text { situations et plus au cours des } \\
3 \text { dernières années) et taux } \\
\text { élevé d'absentéisme. }\end{array}$ \\
\hline $\begin{array}{l}\text { L'organisme repose sur } \\
\text { une équipe motivée et } \\
\text { complémentaire qui } \\
\text { s'implique dans le processus } \\
\text { décisionnel }\end{array}$ & $\begin{array}{l}\text { L'organisme repose sur } \\
\text { quelques individus clés qui } \\
\text { s'impliquent dans le processus } \\
\text { décisionnel. }\end{array}$ & $\begin{array}{l}\text { L'organisme repose sur une } \\
\text { seule personne clé qui prend } \\
\text { l'ensemble des décisions. }\end{array}$ \\
\hline $\begin{array}{l}\text { Les employés ont un } \\
\text { plan de formation et de } \\
\text { développement élaboré pour } \\
\text { leurs besoins personnels et } \\
\text { en complémentarité entre les } \\
\text { membres de l'équipe. }\end{array}$ & $\begin{array}{l}\text { Les employés ont un } \\
\text { plan de formation et de } \\
\text { développement sans lien entre } \\
\text { les membres de l'équipe et la } \\
\text { stratégie globale. }\end{array}$ & $\begin{array}{l}\text { Les employés n'ont aucun } \\
\text { plan de formation ou de } \\
\text { développement. }\end{array}$ \\
\hline $\begin{array}{l}\text { Une appréciation annuelle } \\
\text { est réalisée avec les employés } \\
\text { ainsi qu'une rencontre de } \\
\text { progression à la mi-année. }\end{array}$ & $\begin{array}{l}\text { Une appréciation annuelle est } \\
\text { réalisée avec les employés. }\end{array}$ & $\begin{array}{l}\text { Aucune appréciation annuelle } \\
\text { n'est réalisée avec les employés } \\
\text { ou le processus établi } \\
\text { d'appréciation est rarement } \\
\text { complété. }\end{array}$ \\
\hline $\begin{array}{l}\text { La direction/coordination } \\
\text { fait confiance aux membres } \\
\text { de son équipe et délègue } \\
\text { efficacement. }\end{array}$ & $\begin{array}{l}\text { La direction/coordination } \\
\text { délègue inégalement, } \\
\text { beaucoup à certaines } \\
\text { personnes et peu à d'autres. }\end{array}$ & $\begin{array}{l}\text { La direction/coordination } \\
\text { délègue peu ou pas. }\end{array}$ \\
\hline Nombre de points : & Nombre de points : & Nombre de points : \\
\hline
\end{tabular}




\begin{tabular}{|c|c|c|}
\hline \multicolumn{2}{|c|}{ STRATÉGIE ET PLANIFICATION } & \multirow{2}{*}{$\begin{array}{l}\text { Total de points : } \\
\text { (max. } 21 \text { points) } \\
\text { FAIBLE (1) }\end{array}$} \\
\hline FORT (3) & ADÉQUAT (2) & \\
\hline $\begin{array}{l}\text { L'organisation a une } \\
\text { planification stratégique } \\
\text { sur trois ou cinq ans qui } \\
\text { reflète bien la mission et les } \\
\text { intérêts de l'organisme et le } \\
\text { développement qu'elle désire } \\
\text { faire. }\end{array}$ & $\begin{array}{l}\text { L'organisation a une } \\
\text { planification annuelle. }\end{array}$ & $\begin{array}{l}\text { L'organisation ne planifie pas } \\
\text { ou très peu. }\end{array}$ \\
\hline $\begin{array}{l}\text { Les actions/activités de } \\
\text { l'organisme sont axées sur la } \\
\text { planification et vit peu ou pas } \\
\text { de situations de crise. }\end{array}$ & $\begin{array}{l}\text { L'organisme suit une } \\
\text { planification et vit des crises } \\
\text { régulièrement. }\end{array}$ & $\begin{array}{l}\text { L'organisme est axé sur les } \\
\text { urgences. Éteint des feux } \\
\text { continuellement. }\end{array}$ \\
\hline $\begin{array}{l}\text { L'organisme suit sa stratégie } \\
\text { et sa planification et priorise } \\
\text { efficacement. }\end{array}$ & $\begin{array}{l}\text { L'organisme suit sa } \\
\text { planification. }\end{array}$ & $\begin{array}{l}\text { L'organisme ne suit pas sa } \\
\text { planification ou n'a aucune } \\
\text { planification. }\end{array}$ \\
\hline $\begin{array}{l}\text { L'organisme a établi des cycles } \\
\text { de travail annuel (tâches } \\
\text { récurrentes mensuellement et } \\
\text { annuellement) afin de faciliter } \\
\text { l'organisation du travail ainsi } \\
\text { que gérer efficacement les } \\
\text { priorités. (temps de demandes } \\
\text { de subvention, évaluations, } \\
\text { campagnes, recrutement de } \\
\text { bénévoles, AGA). }\end{array}$ & $\begin{array}{l}\text { L'organisme à une idée } \\
\text { générale du travail annuelle et } \\
\text { y-va par priorité. }\end{array}$ & $\begin{array}{l}\text { L'organisme n'a pas de vue } \\
\text { d'ensemble du travail annuel et } \\
\text { des priorités. }\end{array}$ \\
\hline $\begin{array}{l}\text { Les rencontres d'équipe et avec } \\
\text { les partenaires sont régulières, } \\
\text { dynamiques et efficaces. }\end{array}$ & $\begin{array}{l}\text { Les rencontres avec l'équipe et } \\
\text { les partenaires sont régulières. }\end{array}$ & $\begin{array}{l}\text { L'organisme souffre de } \\
\text { réunionites (beaucoup de } \\
\text { réunions, peu d'avancement) } \\
\text { ou ne fait pas de réunions. }\end{array}$ \\
\hline $\begin{array}{l}\text { Il est possible de trouver un } \\
\text { document dans l'organisation } \\
\text { en moins de } 5 \text { minutes. }\end{array}$ & $\begin{array}{l}\text { Il faut chercher plus de } 15 \\
\text { minutes pour trouver un } \\
\text { document que l'on cherche au } \\
\text { sein de l'organisation. }\end{array}$ & $\begin{array}{l}\text { L'organisation sait vaguement } \\
\text { où sont les choses et les } \\
\text { documents. }\end{array}$ \\
\hline $\begin{array}{l}\text { Organisation promet moins et } \\
\text { livre plus. }\end{array}$ & $\begin{array}{l}\text { Organisation promet beaucoup } \\
\text { et livre avec beaucoup de stress. }\end{array}$ & $\begin{array}{l}\text { Organisation promet trop et ne } \\
\text { livre pas. }\end{array}$ \\
\hline Nombre de points : & Nombre de points : & Nombre de points : \\
\hline
\end{tabular}




\begin{tabular}{|c|c|c|}
\hline \multicolumn{2}{|l|}{ LEADERSHIP } & \multirow{2}{*}{$\begin{array}{l}\text { Total de points : } \\
\text { (max. } 21 \text { points) } \\
\text { FAIBLE }(1)\end{array}$} \\
\hline FORT (3) & ADÉQUAT (2) & \\
\hline $\begin{array}{l}\text { La direction et/ou coordination } \\
\text { est une source d'inspiration } \\
\text { pour l'équipe et le conseil } \\
\text { d'administration. }\end{array}$ & $\begin{array}{l}\text { La direction et/ou coordination } \\
\text { n'est ni une source } \\
\text { d'inspiration, ni une source de } \\
\text { démotivation. }\end{array}$ & $\begin{array}{l}\text { La direction et/ou coordination } \\
\text { est une source de démotivation. }\end{array}$ \\
\hline $\begin{array}{l}\text { La direction et/ou coordination } \\
\text { est claire dans ses messages. }\end{array}$ & $\begin{array}{l}\text { La direction et/ou coordination } \\
\text { est ambigüe dans ses messages. }\end{array}$ & $\begin{array}{l}\text { La direction et/ou coordination } \\
\text { est vague dans ses messages. }\end{array}$ \\
\hline $\begin{array}{l}\text { La direction et/ou coordination } \\
\text { livre ses engagements dans des } \\
\text { délais raisonnables. }\end{array}$ & $\begin{array}{l}\text { La direction et/ou coordination } \\
\text { complète ses promesses, mais } \\
\text { avec beaucoup de temps } \\
\text { d'attente. }\end{array}$ & $\begin{array}{l}\text { La direction et/ou coordination } \\
\text { ne tient pas ses promesses. }\end{array}$ \\
\hline $\begin{array}{l}\text { La direction et/ou } \\
\text { coordination fait face aux } \\
\text { situations rapidement, même } \\
\text { inconfortables, avant que cela } \\
\text { devienne des problèmes. }\end{array}$ & $\begin{array}{l}\text { La direction et/ou } \\
\text { coordination finit par usure à } \\
\text { adresser des situations qui sont } \\
\text { devenues inconfortables ou } \\
\text { problématiques. }\end{array}$ & $\begin{array}{l}\text { La direction et/ou coordination } \\
\text { ignore des situations qui } \\
\text { finissent par devenir des crises. }\end{array}$ \\
\hline $\begin{array}{l}\text { La direction et/ou coordination } \\
\text { modèle le comportement } \\
\text { préconisé au sein de } \\
\text { l'organisme. (Les bottines } \\
\text { suivent les babines!) }\end{array}$ & $\begin{array}{l}\text { La direction et/ou coordination } \\
\text { modèle, par vagues, le } \\
\text { comportement préconisé } \\
\text { au sein de l'organisme. (Les } \\
\text { bottines suivent parfois les } \\
\text { babines!) }\end{array}$ & $\begin{array}{l}\text { La direction et/ou coordination } \\
\text { est en contradiction avec le } \\
\text { comportement préconisé } \\
\text { au sein de l'organisme. (Les } \\
\text { bottines ne suivent pas les } \\
\text { babines!) }\end{array}$ \\
\hline $\begin{array}{l}\text { La direction et/ou coordination } \\
\text { fait preuve d'une grande écoute } \\
\text { et ouverture. Elle est accessible } \\
\text { aux membres de l'organisme } \\
\text { (porte du bureau ouverte) }\end{array}$ & $\begin{array}{l}\text { La direction et/ou coordination } \\
\text { écoute par vagues et fait preuve } \\
\text { d'un peu d'ouverture. }\end{array}$ & $\begin{array}{l}\text { La direction et/ou coordination } \\
\text { n'écoute pas et est très peu } \\
\text { ouverte. }\end{array}$ \\
\hline $\begin{array}{l}\text { La direction et/ou coordination } \\
\text { est proactive et passe à l'action } \\
\text { rapidement pour transformer } \\
\text { les choses. }\end{array}$ & $\begin{array}{l}\text { La direction et/ou coordination } \\
\text { est active et attend que les } \\
\text { situations se corsent avant de } \\
\text { passer à l'action et d'agir. }\end{array}$ & $\begin{array}{l}\text { La direction et/ou coordination } \\
\text { est réactive et attends } \\
\text { longtemps avant de passer à } \\
\text { l'action. }\end{array}$ \\
\hline Nombre de points : & Nombre de points : & Nombre de points : \\
\hline
\end{tabular}




\begin{tabular}{|l|l|l|}
\hline \multicolumn{2}{|l|}{ ATTITUDES de l'équipe } & $\begin{array}{l}\text { Total de points : } \\
\text { (max. 21 points) }\end{array}$ \\
\hline FORT (3) & ADÉQUAT (2) & FAIBLE (1) \\
\hline $\begin{array}{l}\text { Les membres de l'équipe ont } \\
\text { une attitude d'ouverture et de } \\
\text { compassion. }\end{array}$ & $\begin{array}{l}\text { Les membres de l'équipe } \\
\text { ont une attitude de } \\
\text { compréhension et de } \\
\text { tolérance. }\end{array}$ & $\begin{array}{l}\text { Les membres de l'équipe ont } \\
\text { une attitude de supériorité et } \\
\text { de pitié. }\end{array}$ \\
\hline $\begin{array}{l}\text { L'équipe met l'accent sur les } \\
\text { forces. }\end{array}$ & $\begin{array}{l}\text { L'équipe met l'accent sur les } \\
\text { faiblesses et les lacunes. }\end{array}$ & $\begin{array}{l}\text { L'équipe met l'accent sur les } \\
\text { faiblesses, les lacunes et les } \\
\text { insatisfactions. }\end{array}$ \\
\hline $\begin{array}{l}\text { L'équipe met l'accent sur } \\
\text { les possibilités et trouve des } \\
\text { solutions. }\end{array}$ & $\begin{array}{l}\text { L'équipe met l'accent sur } \\
\text { les limites et trouve des } \\
\text { problèmes. }\end{array}$ & $\begin{array}{l}\text { L'équipe met l'accent sur les } \\
\text { excuses et les justifications. }\end{array}$ \\
\hline $\begin{array}{l}\text { Les membres de l'équipe } \\
\text { reconnaissent, encouragent et } \\
\text { soulignent la progression. }\end{array}$ & $\begin{array}{l}\text { Les membres de l'équipe } \\
\text { voient uniquement ce qui } \\
\text { reste à faire. }\end{array}$ & $\begin{array}{l}\text { Les membres de l'équipe se } \\
\text { plaignent de ce qui reste à } \\
\text { faire. }\end{array}$ \\
\hline $\begin{array}{l}\text { L'équipe fait preuve d'une } \\
\text { attitude d'espoir, de passion et } \\
\text { de gratitude. }\end{array}$ & $\begin{array}{l}\text { L'équipe fait preuve d'une } \\
\text { attitude de frustration, } \\
\text { d'impatience, de colère ou de } \\
\text { désintéressement. }\end{array}$ & $\begin{array}{l}\text { L'équipe fait preuve d'une } \\
\text { attitude de découragement, de } \\
\text { désespoir et de défaitisme. }\end{array}$ \\
\hline $\begin{array}{l}\text { Il y a un esprit d'échange, } \\
\text { d'ouverture et de se dire } \\
\text { les choses dans l'équipe et } \\
\text { l'organisation. }\end{array}$ & $\begin{array}{l}\text { Après beaucoup de } \\
\text { commérages et d'inconfort, } \\
\text { les choses finissent par venir } \\
\text { à la surface dans l'équipe et } \\
\text { l'organisation. }\end{array}$ & $\begin{array}{l}\text { Il y a beaucoup de } \\
\text { commérages dans l'équipe et } \\
\text { l'organisation. }\end{array}$ \\
\hline Excellente ambiance de travail. & Bonne ambiance de travail. & Ambiance de travail lourde. \\
\hline Nombre de points : & Nombre de points : & Nombre de points : \\
\hline
\end{tabular}




\begin{tabular}{|c|c|c|}
\hline \multicolumn{3}{|c|}{$\begin{array}{c}\text { SYNTHÈSE DES POINTS } \\
\text { Faites le total des résultats dans chaque catégorie. } \\
\text { Inscrivez le résultat de chaque catégorie dans le tableau ci-dessous. }\end{array}$} \\
\hline CATÉGORIES & $\begin{array}{c}\text { LES RÉSULTATS DE LA } \\
\text { SECTION }\end{array}$ & MAXIMUM POSSIBLE \\
\hline Mission et valeurs & & 21 \\
\hline Vie associative & & 21 \\
\hline Conseil d'administration & & 21 \\
\hline $\begin{array}{l}\text { Ancrage dans le milieu et } \\
\text { réseautage }\end{array}$ & & 21 \\
\hline Santé financière & & 21 \\
\hline Pratiques & & 21 \\
\hline Ressources humaines & & 21 \\
\hline Stratégie et planification & & 21 \\
\hline Leadership & & 21 \\
\hline Attitudes & & 21 \\
\hline Total & & 210 \\
\hline
\end{tabular}




\section{GRAPHIQUE DE RÉSULTATS}

Pour chaque section, noircissez le total des résultats.

\begin{tabular}{|c|c|c|c|c|c|c|c|c|c|c|}
\hline 21 & & & & & & & & & & \\
\hline 20 & & & & & & & & & & \\
\hline 19 & & & & & & & & & & \\
\hline 18 & & & & & & & & & & \\
\hline 17 & & & & & & & & & & \\
\hline 16 & & & & & & & & & & \\
\hline 15 & & & & & & & & & & \\
\hline 14 & & & & & & & & & & \\
\hline 13 & & & & & & & & & & \\
\hline 12 & & & & & & & & & & \\
\hline 11 & & & & & & & & & & \\
\hline 10 & & & & & & & & & & \\
\hline 9 & & & & & & & & & & \\
\hline 8 & & & & & & & & & & \\
\hline 7 & & & & & & & & & & \\
\hline 6 & & & & & & & & & & \\
\hline 5 & & & & & & & & & & \\
\hline 4 & & & & & & & & & & \\
\hline 3 & & & & & & & & & & \\
\hline 2 & & & & & & & & & & \\
\hline 1 & & & & & & & & & & \\
\hline 0 & & & & & & & & & & \\
\hline & 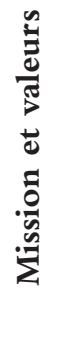 & 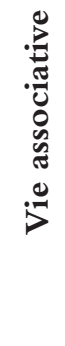 & 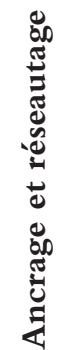 & 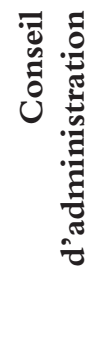 & 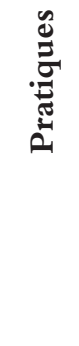 & 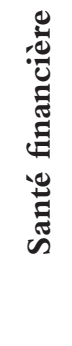 & 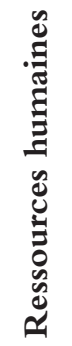 & 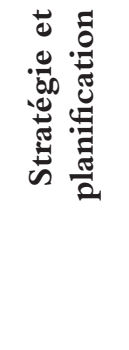 & 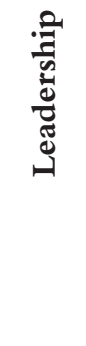 & $\underbrace{\stackrel{0}{Z}}_{\stackrel{D}{Z}}$ \\
\hline
\end{tabular}

